\title{
DEVELOPMENT INTEGRATED INDUCTIVE SENSORS FOR MAGNETIC IMMUNOASSAY IN "LAB ON CHIP" DEVICES
}

\author{
Bruno Andò, Salvatore Baglio, \\ Angela Beninato, Vincenzo Marletta \\ D.I.E.E.S., University of Catania, Italy \\ angela.beninato@diees.unict.it
}

\author{
Giorgio Fallica \\ STMicroelectronics, Catania, Italy \\ giogio.fallica@st.com
}

\begin{abstract}
In this paper an inductive integrated sensor to be adopted in sensing application on microfluidic systems for biomedical applications is presented. It is based on the use of magnetic particles that, suitably coated, act as markers of the bio-molecule to be detected. The device consists of a primary coil and two secondary coils arranged in a differential configuration. The sensing principle of the device is related to the output voltage variation on the secondary coils due to the presence of the magnetic beads only over one of the two coils. A general transduction mechanism is therefore presented together with analytical models and some discussion on novel integrated devices whose layout is proposed here. These sensors will be embedded into a more general "lab on a chip" device in which suitable microfluidics will be implemented both to bind markers with analyte and to drive the fluid into the sensing chamber.
\end{abstract}

\section{Key words}

Magnetic immuno-assay, magnetic beads, inductive sensors, lab-on-chip.

\section{Introduction}

In the modern research the realization of biosensors for applications in different fields like public health, clinical analysis, water and air pollution, biotechnology holds an important position. For such devices a very high sensitivity and specificity, a short analysis time, low cost, easiness to handle, capability for portable applications are of fundamental importance. Biosensors with high sensitivity and specificity can be obtained by using Immunoassay techniques [Larsson et al. 1999], [Kriz et al. 1998], which is a biochemical test that measures the concentration of a substance in a biological liquid using the reaction of an antibody or antibodies to its antigen. Detection is made by coupling these molecules to suitable markers, such as enzymes, radioactive compounds, fluorophores, luminescence and magnetic ones. By comparison with the other, magnetic markers have potential advantages, which are related to their low price, very high stability and absence of toxicity. In addition, biomolecules fixed to magnetic nanoparticles can be easily localized and manipulated by suitable magnetic fields [Kriz et al. 1998],[Baselt et al., 1997],[Edelstein et al., 2000]. The problem of detecting biological agents is therefore shifted to the ability of sensing the presence of the chosen marker and, in particular, referring to the magnetic markers the issue arising concerns the possibility of detecting the weak magnetic field, or perturbation to an externally applied magnetic field, due to the presence of a small number of particles having magnetic properties. In Fig.1 the detection principle is shown.

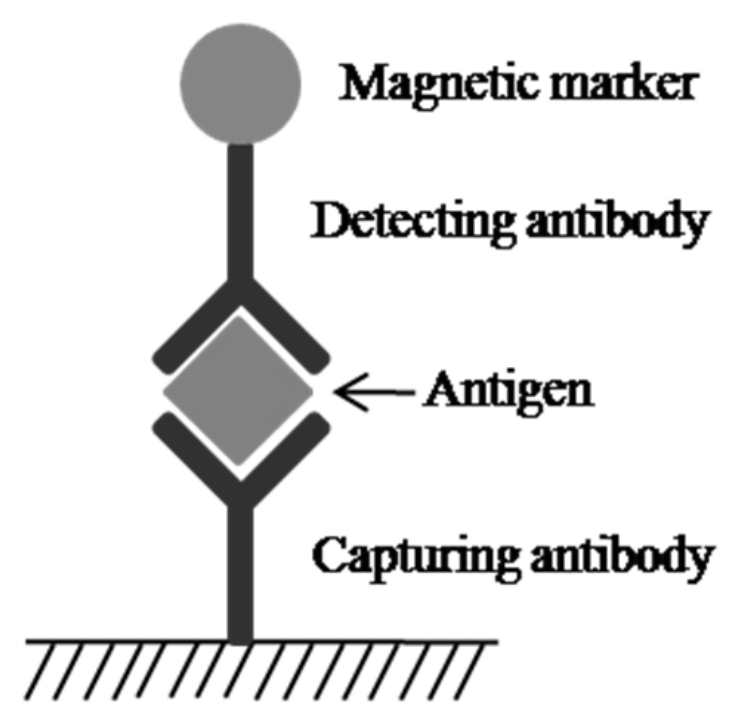

Figure 1. Bond antibody-antigen-antibody-marker in the magnetic immunoassay.

several different works have been presented in the scientific literature which deal with the problem of detect- 
ing the presence of magnetic markers into a selected area, [Baglio et al. 2005], [Serre et al. 2006], [Baglio et al. 2007], [Azimi et al. 2007], [Vilà et al. 2005]: in these sensors the inductance value changes as function of a certain density of magnetic particles in the active region of the sensor device. First the active region must be functionalized, very often silanizated, to allow the binding of the antibody over the sensor surface; then both the active region and the magnetic particles must be functionalized with a antibody specific to the analyte to be detected. The interaction antibody-analyteantibody binds the magnetic particles to the sensors surface. if a magnetic field source is considered, the distribution of the magnetic flux lines will be affected by the presence of a number of magnetic particles, this in turn induces a change in the inductive impedance of the coil placed in the active area of the sensor.

In comparison with other kinds of magnetic biosensors, inductive devices have several potential advantages, which are related to their higher simplicity, fully compatibility with standard Si technology materials, low cost and higher flexibility.

In this work the inductive sensor used to measure the amount of magnetic particles bounded to the sensor surface will be presented together with its analytical model, then some technology related issues will be discussed and the layout of some novel inductive devices for magnetic immunoassay will be presented. These layout refer to more rich devices where an on-chip temperature control is planned. In fact both the heater and the temperature sensor will be embedded into the same device.

Finally, a brief discussion on the complete system envisaged for the lab on chip magnetic immunoassay device will be presented together with some conclusive remarks.

\section{THE INDUCTIVE SENSOR}

The device presented here is based on a planar coreless differential transformer configuration. A primary coil generates a magnetic flux which links with two secondary coils, having opposite winding sense, connected in a differential arrangement. The primary coil generates a magnetic flux that therefore induces voltages with equal values but opposite in sign in the secondary coils, due to their opposite winding sense and the symmetry properties of the device; therefore the resulting output voltage, which is the difference between the voltages across the secondary coils, is zero when no magnetic particles are present. On the other hand, the presence of magnetic particles in one of the secondary coils will cause an increase of the magnetic field density on a area close to the magnetic particles; in this way, the output voltage will be non-zero in fact the voltages induced into the two secondary windings will not be equal anymore. This working principle is schematized in Fig.2. In the proposed device only the secondary coil over which the magnetic particles are
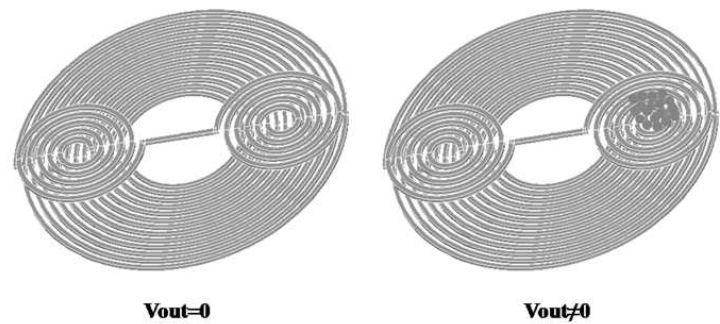

Figure 2. Working principle of the planar differential transformer.

placed, acts as the "active" sensor, while the other one acts as "dummy", like in most differential sensing approaches. In particular here the differential configuration is used not to enhance sensitivity; in fact there are no opposite variations of inductance, but to lower the noise floor. In fact any unwanted external excitation will affect the two secondary coils at the same manner and therefore will be nulled by the differential arrangement, while only the signal produced by the magnetic markers placed over one of the two coils will be useful for a non zero output voltage.

The primary coil is a source of excitation of the sensor. This approach allows a more flexible optimization of the device in terms of sensitivity; in fact in the case in which the primary coil is current driven and the secondary coil has an infinite impedance, the open circuit voltage at the secondary coil, if expressed in terms of the current applied to the primary winding, is proportional to the product of the number of turns of the primary and the secondary coils as reported in Equation (1)

$$
S=N_{1} N_{2} \frac{2 \Re_{1}+\Re_{2}}{\left[\Re_{1}\left(2-\frac{\Delta L}{L_{2}^{\prime}}\right)+\Re_{2}\left(1-\frac{\Delta L}{L_{2}^{\prime}}\right)\right]^{2}} \frac{d i_{1}}{d t}
$$

Where:

- $N_{1}$ represents the number of turns of the primary coil;

- $N_{2}$ represents the number of turns of the secondary coil;

- $\Re_{1}$ and $\Re_{2}$ are the reluctances of primary and secondary coils;

- $i_{1}$ is the current in the primary coil;

- $\Delta L$ is the increase of inductance value of the secondary coil over which the magnetic beads are placed due to the increase in the magnetic flux;

- $L_{2}^{\prime}$ is the inductance value of the secondary coil over which the magnetic beads are placed.

This differential transformer is realized in a dedicated technology based on STMicroelectronics fabrication facilities. It is made up of two metal layers (Metal1 and Metal2) separated by a layer of TEOS oxide. The primary winding has been realized in the Metal1 layer, while the Metal2 has been used to realize the sec- 
ondary windings. The device was studied and optimized in function of the geometric parameters [Ando' et al. 2009] such to determine the optimal dimension of both the primary and secondary coils and the optimal relative position. This optimization process has been performed taking into account the technology parameters of the dedicated process designed for this project. Particular efforts, related to this latter issue, have been payed to the top surface quality that must be suitable to the antibody binding necessary for realizing the immunoassay process, therefore planarization has been considered together with the deposition of a final thin layer of gold over the contact pad area in order to protect contacts from the further surface silanization.

Some of these device layouts are shown in Fig.3. In particular in Fig.3a the basic device, made up of two primary coils superimposed with two secondary coils is shown. Only one side of the secondary windings is functionalized to bind the antibody. Moreover a twofold detection strategy is implemented: a larger coils is used to detect and quantify the amount of the magnetic particles while the smaller one will be used to detect the target presence (ideally it is aimed to sense the presence of the "single" magnetic bead).

In Fig.3c, the differential mechanism has been implemented here by designing a couple of identical superimposed windings and then by connenting the secondary coils in a counterphased arrangment.

In order to adequately perform the immunoassay protocol an accurate temperature control is required, therefore both a heater and a temperature sensor have been embedded into the proposed devices.

This has required the introduction of a high resisitivithy polysilicon layer into the process (actually an additive photolitographic masking step to obtain different conductinties into the deposited polysilicon layer) to realize the resistive heater. The resistive heater has been designed to uniformely cover the region below the two secondary coils.

In order to control the temperature suitable sensors have been considered. Given the materials avalable, two types of sensors have been designed: a RTD thermoresistor (Fig.3a-c) and a thermopile, series of 7 thermocouples, made by using the Metall and the Poly layers (Fig.3b). In this latter case the RTD has been inserted only for comparison reasons.

\section{THE LAB ON CHIP DEVICE}

This inductive sensor implements the sensing strategy of a more general device, that can be summarized as in Fig.4. The functionalized magnetic beads and the fluid sample containing the analyte bounded to the antibodies, are driven into the "mixing chamber" to realize the antibody-antigene binding schematized in the upper section of the sandwich shown in Fig.1. This agglomerate is driven to the "sensing chamber" by a valve-pump system. If the chambers are pre-loaded or, a positive pressure difference is applied, valves can be single-use

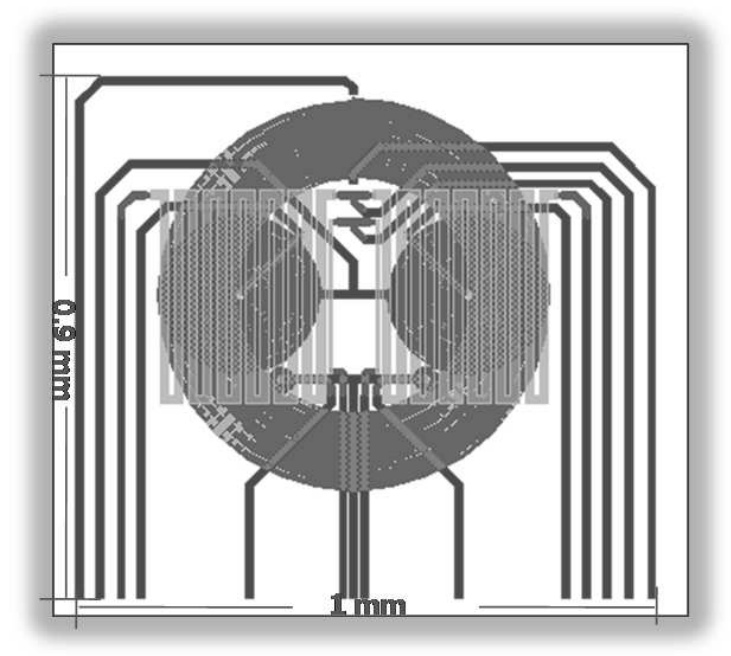

(a)

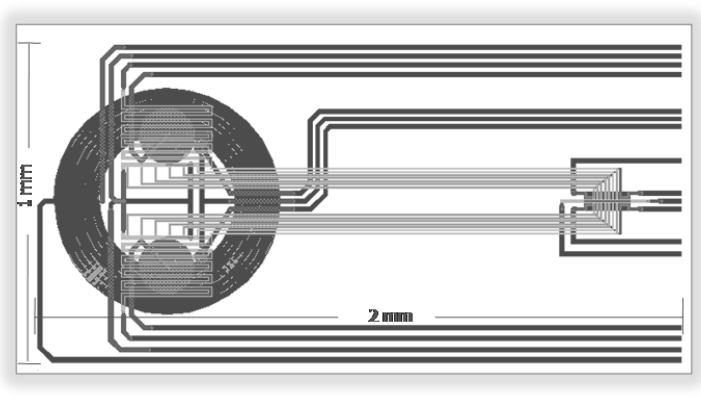

(b)

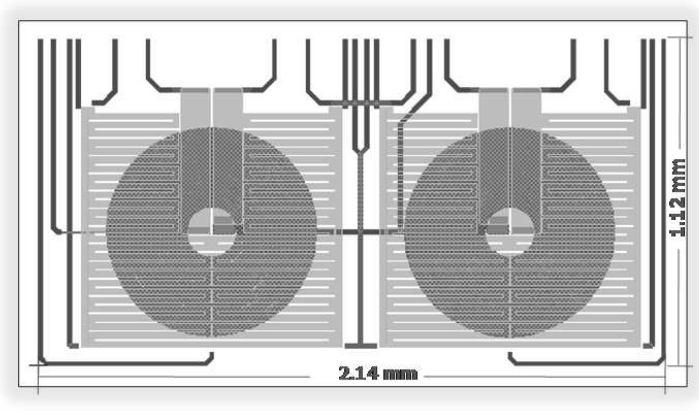

(c)

Figure 3. Three optimized device layouts of the differential inductive sensor for immunoassay with magnetic beads. (a) Differential transformer with dual secondary winding for both "single bead" and "average" detection, resistive heater and RTD temperature monitoring; (b) Differential transformer with the thermopile as temperature sensor; (c) Dual transformer topology with differential connetion of the output terminals, resistive heater and RTD temperature sensor.

because the sample goes from the "mixing chamber" to the "sensing chamber"; otherwise a volumetric pumping mechanism can be adopted to move the fluid to the "sensing chamber" without the need of valves.

The inductive sensor will be placed on the bottom of the "sensing chamber" and here the immunoassay mechanism will be completed by the binding of the 


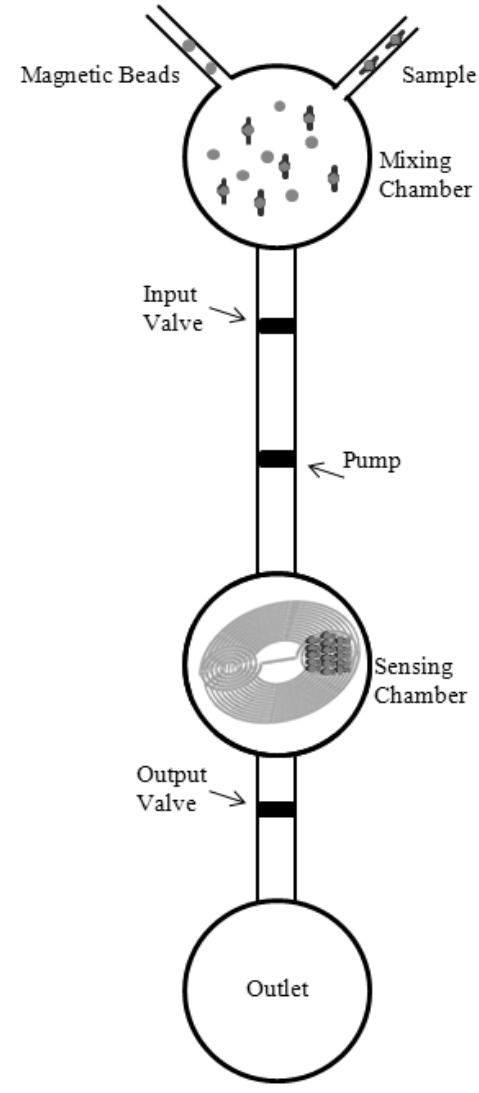

Figure 4. The functional block scheme of the lab on chip system embedding the inductive sensor for magnetic beads.

functionalized magnetic beads to the receptors over the active sensor surface to complete the sandwich shown in Fig.1. In the "sensing chamber" the above described sensor reveals the amount of the molecule to detect.

\section{CONCLUSION}

In this paper an inductive sensors for applications to magnetic immuno-assay in microfluidic systems has been presented. In particular here the fabrication process and the layout of three novel devices have been discussed. The devices here embed also a temperature monitoring solution that has been realized with the materials available in the dedicated fabrication process.

The focus here is on the optimal design of the inductive transducers but the global goal, and work is in progress in this direction, is toward integrated microfluidic systems in lab-on-a-chip scenario.

\section{References}

K. Larsson, K. Kriz, D. Kriz, (1999). Magnetic Transducers in Biosensors and Bioassays. Analusis, 27(7), pp. 617-621.

K. Kriz, J. Gehrke, D. Kriz (1998). Advance toward magneto immunoassays. Biosensors and Bioelec., 13, pp. 817-821.

D.R.Baselt, G.U.Lee, K.M.Handen, L.A.Chrisey and
R.J.Colton (1997). High-sensitivity Micromachined Biosensor. Proc. of the IEEE, 85, pp. 672.

R.L.Edelstein, C.R.Tamanaha, P.E.Sheehan, M.M.Miller, D.R.Baselt, L.J.Whitman and R.J.Colton (2000) The BARC biosensor applied to the detection of biological warfare agents. Biosensors and Bioelectronics, 14, pp. 805.

S. Baglio, S. Castorina, . N. Savalli (2005). Integrated inductive sensors for magnetic immuno assay applications. IEEE Sensors Journal, 5(3), pp. 372-384.

C. Serre, S. Martnez, A.Pérez-Rodrìguez, J.R. Morante, J.Esteve, J.Montserrat (2006) Si technology based microinductive devices for biodetection applications. Sensors and Actuators A, 132(3), pp. 499505.

S. Baglio, A. Pérez-Rodrìguez, S. Martnez, C. Serre, J.R. Morante, J. Esteve, J. Montserrat (2007) Microinductive Signal Conditioning with Resonant Differential Filter: High Sensitivity Biodetection Applications. IEEE transaction Instrumentation and Measurements, 56(5), pp. 1590-1595.

S.M. Azimi, M.R. Bahmanyar, M.Zolgharni, and W.Balachandran (2007). An Inductance-based Sensor for DNA Hybridization Detection. In Proc. of the 2nd IEEE International Conference on Nano/Micro Engineered and Molecular Systems.

A. Vilà, A. Romano-Rodrìguez, F. Hernàndez, S. Martìnez, C. Serre, A. Pèrez-Rodrguez, J.R. Morante (2005) Microcoils for biosensors fabricated by focused ion beam (FIB). Electron Devices, 56, pp. 209212.

Bruno Andò, Salvatore Baglio, Angela Beninato, Giorgio Fallica, Vincenzo Marletta, Nicola Pitrone, (2009). Analysis and design of inductive biosensors for magnetic immunoassay. In Proc. of the XIX IMEKO World Congress Fundamental and Applied Metrology. Lisbon, Portugal. 